\title{
Graph Neural Architecture Search
}

\author{
Yang Gao ${ }^{1,5}$, Hong Yang ${ }^{2 *}$, Peng Zhang ${ }^{3}$, Chuan Zhou ${ }^{4,5 *}$ and Yue Hu ${ }^{1,5}$ \\ ${ }^{1}$ Institute of Information Engineering, Chinese Academy of Sciences, Beijing, China \\ ${ }^{2}$ Centre for Artificial Intelligence, University of Technology Sydney, Australia \\ ${ }^{3}$ Ant Financial Services Group, Hangzhou, China \\ ${ }^{4}$ Academy of Mathematics and Systems Science, Chinese Academy of Sciences, Beijing, China \\ ${ }^{5}$ School of Cyber Security, University of Chinese Academy of Sciences, Beijing, China \\ gaoyang@iie.ac.cn, hong.yang@student.uts.edu.au,zhangpeng04@gmail.com, \\ zhouchuan@amss.ac.cn, huyue@iie.ac.cn
}

\begin{abstract}
Graph neural networks (GNNs) emerged recently as a powerful tool for analyzing non-Euclidean data such as social network data. Despite their success, the design of graph neural networks requires heavy manual work and domain knowledge. In this paper, we present a graph neural architecture search method (GraphNAS) that enables automatic design of the best graph neural architecture based on reinforcement learning. Specifically, GraphNAS uses a recurrent network to generate variable-length strings that describe the architectures of graph neural networks, and trains the recurrent network with policy gradient to maximize the expected accuracy of the generated architectures on a validation data set. Furthermore, to improve the search efficiency of GraphNAS on big networks, GraphNAS restricts the search space from an entire architecture space to a sequential concatenation of the best search results built on each single architecture layer. Experiments on real-world datasets demonstrate that GraphNAS can design a novel network architecture that rivals the best human-invented architecture in terms of validation set accuracy. Moreover, in a transfer learning task we observe that graph neural architectures designed by GraphNAS, when transferred to new datasets, still gain improvement in terms of prediction accuracy.
\end{abstract}

\section{Introduction}

Graph neural networks (GNNs) emerged recently as a powerful tool for analyzing non-Euclidean data such as social network data. The applications of GNNs span over online recommendation [Wu et al., 2019b], traffic forecasting [Yu et al., 2018] and action recognition [Yan et al., 2018]. The basic idea of GNNs is to propagate feature information between neighboring nodes so that nodes can learn feature representations by using the locally connected graph architecture information. Popular GNNs include but not limited to GCN [Kipf and Welling, 2017], GraphSAGE [Hamilton et al., 2017],

\footnotetext{
${ }^{*}$ Corresponding authors
}

GAT [Velickovic et al., 2017] and APPNP [Klicpera et al., 2019].

Despite the success of GNNs, the design of graph neural architectures requires both heavy manual work and domain knowledge. Similar to CNNs that contain many manual parameters such as the size of filters and the type of pooling layers, the results of GNNs heavily rely on the graph neural architectures including the receptive fields, message functions and aggregation functions.

Reinforcement learning has been successfully used to automatically design neural architectures for CNNs and RNNs. The pioneer model NAS [Zoph and Le, 2016] uses a recurrent network as the controller to generate network descriptions of CNNs and RNNs which are referred to as child networks, and then uses validation results of the child networks as reward of the controller to maximize the expected accuracy of the generated architectures of the CNNs and RNNs. According to their experiment results, the NAS search algorithm can improve CNNs and RNNs by a percentage of 0.09 on CIFAR-10 and 3.6 perplexity on the Penn Treebank dataset. Several new neural architecture search algorithms are proposed to improve the efficiency and accuracy of NAS, such as ENAS [Pham et al., 2018] and ProxylessNAS [Cai et al., 2019]. The promising results of using NAS to find the best neural architectures for CNNs and RNNs motivate to use reinforcement learning to find the best graph neural architectures for GNNs.

In this paper, we present a new graph neural architecture search method (GraphNAS) which can automatically design the best graph neural architecture using reinforcement learning. Specifically, we design a new search space for reinforcement learning that covers the operators from the state-of-theart GNNs, such as GCN, GraphSAGE and GAT. Based on the search space, we use a RNN model as the controller to generate variable-length strings that describe the architectures of graph neural networks, and trains the recurrent network with policy gradient to maximize the expected accuracy of the generated architectures on a validation data set. To analyze big networks, we assume the layers of network architectures are independent and restrict the search space to each single layer. Then, the best results found from each single layer are sequentially concatenated to describe the entire architecture. Experiment results show that GraphNAS always obtains 
better results than the state-of-the-art methods. Experiment results also show that GraphNAS obtains performance improvement in a transfer learning task.

The contributions of the paper are summarized as follows:

- This is the first effort to study the challenging problem of using reinforcement learning to design the best graph neural architecture.

- We present a new model GraphNAS to enable the automatic search of the best graph neural architecture, where a new search space is designed that covers the operators from the state-of-the-art GNNs, and a policy gradient algorithm is used to iteratively solve the problem.

- We test GraphNAS on real-world datasets. The results show that our method is capable of designing graph neural architectures that outperform the best humaninvented architectures in terms of validate set accuracy. We have released the python codes on Github ${ }^{1}$ for comparison.

\section{Related Work}

\subsection{Neural Architecture Search (NAS)}

NAS has been popularly used to design convolutional architectures [Zoph and Le, 2016; Pham et al., 2018; Xie et al., 2019; Bello et al., 2017; Liu et al., 2018a; Cai et al., 2019]. The basic idea of NAS is to use reinforcement learning to find the best neural architectures. Specifically, NAS uses a recurrent network to generate architecture descriptions of CNNs and RNNs. Based on NAS, evolution-based NAS [Real et al., 2018] is proposed to use evolution algorithms to simultaneously optimize topology alongside with parameters. ENAS [Pham et al., 2018] allows the sharing of parameters among child models, which enables the search speed 1000 times faster than the standard NAS and obtains a new convolution architecture in 0.45 GPU days. DARTS [Liu et al., 2018a] formulates the task in a differentiable manner which shortens the search of high-performance convolution architectures within four GPU days. Following DARTS [Liu et al., 2018a], GDAS [Dong and Yang, 2019] enables the search speed in four GPU hours, and Proxyless NAS [Cai et al., 2019] claims that the search process can directly operate on the large-scale target tasks and the target hardware platforms. Due to NASbased search algorithms achieve promising results for designing new architectures for CNNs and RNNs, we extend NAS to design graph neural architectures for GNNs in this paper.

\subsection{Graph Neural Networks (GNNs)}

GNNs are firstly discussed in the work [Gori et al., 2005]. Convolutions of GNNs can be categorized into two groups, spectral-based [Kipf and Welling, 2017; Defferrard et al., 2016; Bianchi et al., 2019] and spatial-based [Velickovic et al., 2017; Hamilton et al., 2017; Niepert et al., 2016; You et al., 2019]. Spectral-based convolutions usually handle an entire graph, which is difficult to parallel and hardly scale to big graphs. In contrast, spatial-based convolutions aggregate feature information between neighboring nodes. Spatialbased graph neural architectures mainly consist of three types

\footnotetext{
${ }^{1}$ https://github.com/GraphNAS/GraphNAS
}

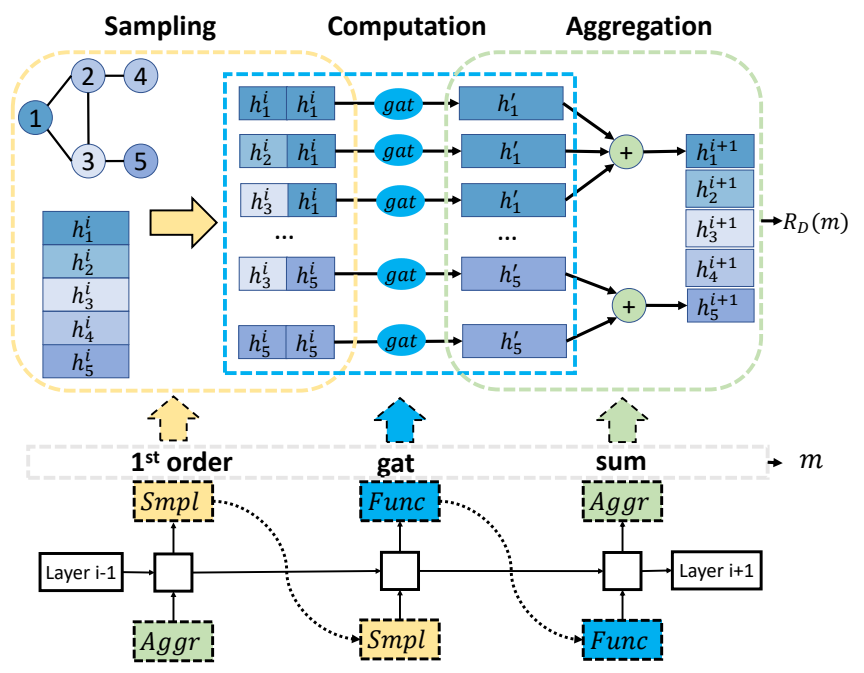

Figure 1: An illustration of GraphNAS. A recurrent network (Controller RNN) generates descriptions of graph neural architectures (child model GNNs). Once an architecture $m$ is generated by the controller, GraphNAS trains $m$ on a given graph $G$ and test $m$ on a validate set $D$. The validation result $R_{D}(m)$ is taken as the reward of the recurrent network.

of operators, i.e., neighbor sampling, message computation and information aggregation. Each layer of the architecture includes the combination of these operators. In this paper, we use reinforcement learning to search the best combination of the operators, instead of manually setting them as in the existing work.

\section{Methods}

In this section, we first formulate the problem of graph neural architecture search with reinforcement learning. Then, we introduce a new search space that covers the operators of the state-of-the-art GNNs. Next, we discuss the search algorithm based on policy gradient. At the last part, we discuss how to extend the search algorithm to big networks.

\subsection{Problem Formulation}

Given a search space of a graph neural architecture $\mathcal{M}$, and a validation set $D$, we aim to find the best architecture $m^{*} \in$ $\mathcal{M}$ that maximizes the expected accuracy $\mathbb{E}\left[\mathcal{R}_{D}(m)\right]$ on the validate set $D$, i.e.,

$$
m^{*}=\arg \max _{m \in \mathcal{M}} \mathbb{E}\left[R_{D}(m)\right] .
$$

Figure 1 shows the reinforcement learning framework used to solve Eq.(1) by continuously sampling architectures $m \in$ $\mathcal{M}$ and evaluating the accuracy (reward) $R$ on the validation set $D$. First, the recurrent network generates a network description $m \in \mathcal{M}$ that corresponds to a GNN model. Then, the generated model $m$ is trained on a given graph $G$ and tested on the validate set $D$. The test result is taken as a reward signal $R$ to update the reinforcement learning.

\subsection{Search Space}

As shown in Figure 1, we use a controller to generate the descriptions (architectures) of GNNs. The controller is a re- 
current neural network with a search space. Similar to the search space of CNNs, each layer of GNNs associates with a search space of the following operators:

1. Neighbor sampling operator Smpl [Hamilton et al., 2017]. This operator selects the receptive field $N(v)$ for a target node $v$. For example, GraphSAGE [Hamilton $e t$ al., 2017] uses sampling to obtain a fixed size of neighbors to handle large graphs.

2. Message computation operator Func [You et al., 2019]. This operator computes the feature information for each node $u$ in the receptive field $N(v)$. Specifically, Func $\left(h_{u}, h_{v}\right)$ can be calculated by $e_{u v} \operatorname{Merg}\left(h_{u}, h_{v}\right)$, where $h_{u}$ and $h_{v}$ are the features of nodes $u$ and $v$ respectively, and $e_{u v}$, as shown in Table 2 , is the correlation coefficient [Velickovic et al., 2017; Liu et al., 2018b; Kipf and Welling, 2017; You et al., 2019] between nodes $u$ and $v$. The Merg operator merges information of nodes $u$ and $v$, such as the CONCAT used in [You et al., 2019; Hamilton et al., 2017].

3. Message aggregation operator Aggr [Hamilton et al., 2017]. This operator aggregates information from the receptive field $N(v)$, which is similar to the pooling operators in CNNs. Any permutation invariant operators, such as mean, max, sum and mlp [Xu et al., 2018] can be used, and non-linear transformations are applied before and/or after the aggregation to achieve accurate expressive power [Zaheer et al., 2017].

4. Multi-head and readout operator Read [Velickovic et al., 2017]. This operator is often used to stabilize the learning process of message computation operators. Similar to the attention mechanism in the work [Vaswani et al., 2017], the multi-head mechanism repeats message computation operator Func for $k$ times with different initializations of Func. Readout operator Read usually includes concatenation and averaging.

Table 1 summarizes the possible values of the above operators. Note that the values are collected from the state-of-theart GNNs, such as GCN, GAT and GraphSAGE. Besides the above operators, we also add extra three operators that are popularly used in CNNs, i.e., the activation function $\sigma$, the number of multi-head $k$ and the output dimension $d$.

We introduce an example of a simple graph neural architecture constructed with the operators given in Table 1. Consider a single layer of GAT with eight heads, 16 hidden units, and an activation function of $e l u$, we describe the architecture by the operators as follows,

$$
\text { [first_order, gat, sum, concat, 8, 16, elu], }
$$

where the first element first_order is an instance of the neighbor sampling operator $S m p l$, the second element gat is the message computation operator Func $=e_{u v}^{g a t} h_{u}$, the third element sum is the message aggregation operator Aggr, the fourth element denotes that the architecture has eight heads, the fifth element denotes that the architecture has 16 hidden units, and the last element denotes $\sigma=e l u$.

For an architecture with $L$ layers, we concatenate the lists of operators built from each layer and generate an architecture

\begin{tabular}{l|l}
\hline Operators & Values \\
\hline Smpl & first_order \\
\hline Func & $e_{u v} h_{u}$ \\
\hline Aggr & sum, mean, max, mlp \\
\hline Read & $\begin{array}{l}\text { avg, for the last layer } \\
\text { concat, otherwise }\end{array}$ \\
\hline \hline activate function $\sigma$ & $\begin{array}{l}\text { sigmoid, tanh, relu, identity, } \\
\text { softplus, leaky_relu, relu6, elu }\end{array}$ \\
\hline multi-head $k$ & $1,2,4,6,8,16$ \\
\hline output dimension $d$ & $8,16,32,64,128,256,512$ \\
\hline
\end{tabular}

Table 1: Operators of search space $\mathcal{M}$

\begin{tabular}{l|l}
\hline$e_{u v}$ & Values \\
\hline const & $e_{u v}^{\text {con }}=1$ \\
\hline gcn & $e_{u v}^{g c n}=1 / \sqrt{d_{u} d_{v}}$ \\
\hline gat & $e_{u v}^{g a t}=$ leaky_relu $\left(\left(W_{l} * h_{u}+W_{r} * h_{v}\right)\right)$ \\
\hline sym-gat & $e_{u v}^{\text {sym }}=e_{v u}^{g a t}+e_{u v}^{g a t}$ \\
\hline cos & $e_{u v}^{c o s}=<W_{l} * h_{u}, W_{r} * h_{v}>$ \\
\hline linear & $e_{u v}^{\text {lin }}=\tanh \left(\operatorname{sum}\left(W_{l} * h_{u}\right)\right)$ \\
\hline gene-linear & $e_{u v}^{g a n}=W_{a} * \tanh \left(W_{l} * h_{u}+W_{r} * h_{v}\right)$ \\
\hline
\end{tabular}

Table 2: Correlation coefficients

of the entire $L$ layers. For example, consider a GNN with two layers. The first layer consists of GCN with 16 hidden units and an activation function relu. The second layer consists of GAT with eight heads, 16 hidden units and an activation function $e l u$. Then, the architecture is described by concatenating the operators of the two layers, which formulates a longer list of operators as follows:

$$
\begin{aligned}
& \text { [ first_order,gcn, sum, concat, } 1,16 \text {, relu } \\
& \text { first_order, gat, sum, avg, } 8,16, \text { elu }] .
\end{aligned}
$$

Because all the operators in the search space $\mathcal{M}$ given in Table 1 are independent, there will be $9408^{L}$ combinations in $\mathcal{M}$, where $L$ is the number of layers. As the search space is too large, we set $L$ to be only two layers in the experiments, which reduces the space to $8.8 \times 10^{7}$. If the architecture is deeper than two layers, a possible solution is to set a timesensitive parameter to control the total search time over the search space.

\subsection{Search Algorithm}

A neural architecture that a controller predicts is a list of operators with length $T$, denoted by $m_{1: T}$, where each operator $m_{i}(1 \leq i \leq T)$ is sampled from the search space $\mathcal{M}$. We use an RNN model parameterized by $\theta$ to generate the operators, as shown at lines 3 to 8 in Algorithm 1 .

In order to maximize the objective function given in Eq.(1), we use a policy gradient algorithm to update parameters $\theta$, so that the controller generates better architectures over time. After the controller generates a list of operators $m_{1: T}$, we build a model $m$ which returns an accuracy of $R_{D}(m)$ on $D$. We use the accuracy as a reward signal to train the controller. Since the reward signal $R$ is non-differentiable, we iteratively update $\theta$ using REINFORCE [Williams, 1992] as follows, 


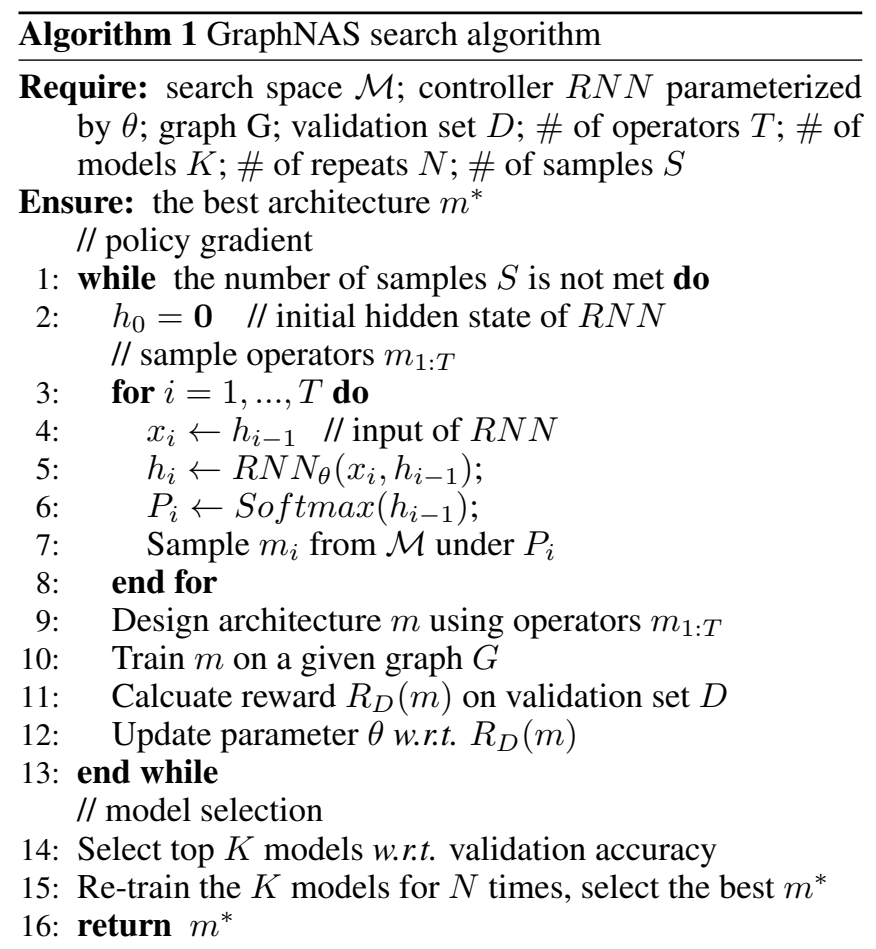

$$
\begin{aligned}
& \nabla_{\theta} \mathbb{E}_{P\left(m_{1: T} ; \theta\right)}[R] \\
& =\sum_{t=1}^{T} \mathbb{E}_{P\left(m_{1: T} ; \theta\right)}\left[\nabla_{\theta} \log P\left(m_{t} \mid m_{t-1: 1} ; \theta\right)(R-b)\right],
\end{aligned}
$$

where $b$ is an exponential moving average of the previous architecture rewards. The training of a child model $m$ is independent of the training of the controller. We choose crossentropy loss function when training $m$. Considering the deviation of the validation accuracy, we select the top $K$ models as candidates and repeatedly train them for $N$ times to reduce variance. The algorithm is summarized in Algorithm 1.

\subsection{Discussions}

What will happen when an architecture goes deeper? Intuitively, the search space of GraphNAS grows exponentially with the number of layers. When the architecture goes deeper, constructing architectures by concatenating the operators will lead to an explosion of the search space. To solve the problem, we enforce three constraints to avoid exponential growth of the search space. First, we assume the layers are independent and design each layer independently. Second, we use domain knowledge of existing GNN architectures and reduce the number of combinations of the operators given in Table 1. Third, We allow the multi-head mechanism using different message computation operators.

In a deeper architecture, how to construct each layer efficiently? Figure 2 gives an example of constructing a single layer by GraphNAS. The layer can be represented as a DAG consisting of two input states $\mathrm{O}_{1}$ and $\mathrm{O}_{2}$, two intermediate states $\mathrm{O}_{3}$ and $\mathrm{O}_{4}$, and one output state $O_{5}$. Each state is a node in the DAG. Specifically, the intermediate states $O_{3}$ and

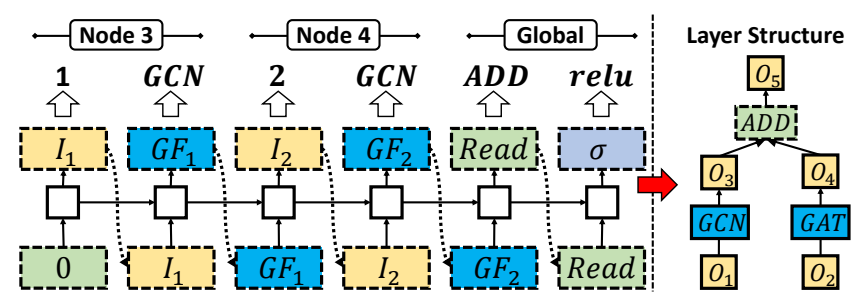

Figure 2: An illustration of GraphNAS constructing a single GNN layer at the right-hand side. The layer has two input states $O_{1}$ and $\mathrm{O}_{2}$, two intermediate states $\mathrm{O}_{3}$ and $\mathrm{O}_{4}$, and an output state $\mathrm{O}_{5}$. The controller at the left-hand side samples $\mathrm{O}_{2}$ from $\left\{\mathrm{O}_{1}, \mathrm{O}_{2}, \mathrm{O}_{3}\right\}$ and take $\mathrm{O}_{2}$ as the input of $\mathrm{O}_{4}$, and then samples $G A T$ for processing $\mathrm{O}_{2}$. The output state $\mathrm{O}_{5}=\operatorname{relu}\left(\mathrm{O}_{3}+\mathrm{O}_{4}\right)$ collects information from $\mathrm{O}_{3}$ and $\mathrm{O}_{4}$, and the controller assigns a readout operator $a d d$ and an activation operator relu for $O_{5}$. As a result, this layer can be described as a list of operators: [1,gcn, 2, gat, add, relu].

$\mathrm{O}_{4}$ are processed by a message computation operator Func, a sample operator $S m p l$, and an aggregation operator $\mathrm{Aggr}$ from its previous state. This procedure of $\mathrm{O}_{4}$ can be formulated as $O_{4}=G A T\left(O_{2}\right)$, where $G A T$ is used to represent the combination of Smpl, Func and Aggr used in GAT. The output state $O_{5}$ is processed by a Read operator from all the intermediate states $\mathrm{O}_{3}$ and $\mathrm{O}_{4}$.

Based on the example, we can generalize the procedure of constructing each layer as follows,

$$
O_{\text {out }}=\sigma\left(\operatorname{Read}\left(O_{i} \mid 3 \leqslant i \leqslant B+2\right)\right),
$$

where $B$ is the number of computation nodes. A controller needs to assign a previous state $O_{j} \in\left\{O_{j} \mid j<i\right\}$, a process operator for all the intermediate states $\left\{O_{i} \mid 3 \leqslant i \leqslant B+2\right\}$, a read-out operator Read, and an activation function $\sigma$ for the output state, as shown at the left-hand side of Figure 2 . We restrict operator Read to be only add, multiply and concat. The operators will cover the following 12 choices:

$\begin{array}{lll}\text { - } \text { identity } & \text { - } 4 \text { head GAT } & \text { - Chebnet } \\ \text { - zeroize } & \text { - } 2 \text { head GAT } & \text { - Mean Sage } \\ \text { - } 8 \text { head GAT } & \text { - } 1 \text { head GAT } & \text { - ARMA } \\ \text { - } 6 \text { head GAT } & \text { - GCN } & \text { - SGC }\end{array}$

where GAT stands for the combination of Smpl, Func and Aggr used in [Velickovic et al., 2017], GCN for [Kipf and Welling, 2017], Chebnet for [Defferrard et al., 2016], Mean Sage for GraphSage[Hamilton et al., 2017] with the mean aggregator, ARMA for [Bianchi et al., 2019], and SGC for [Wu et al., 2019a].

\section{Experiments}

Datasets. We use three popular citation networks, i.e., Cora, Citeseer and Pubmed, as the testbed. To test the capability of transferring the architectures designed by GraphNAS, we use the co-author datasets of MS-CS and MSPhysics, and the product networks of Amazon Computers and Amazon Photos [Shchur et al., 2018]. 


\begin{tabular}{l|ccc|ccc|ccc}
\hline & \multicolumn{4}{|c|}{ Cora } & \multicolumn{3}{c|}{ Citeseer } & \multicolumn{3}{c}{ Pubmed } \\
& semi & sup & rand & semi & sup & rand & semi & sup & rand \\
\hline GCN & $81.4 \pm 0.5$ & $90.2 \pm 0.0$ & $88.3 \pm 1.3$ & $70.9 \pm 0.5$ & $80.0 \pm 0.3$ & $77.2 \pm 1.7$ & $79.0 \pm 0.4$ & $87.8 \pm 0.2$ & $88.1 \pm 1.4$ \\
GAT & $83.0 \pm 0.7$ & $89.5 \pm 0.3$ & $87.2 \pm 1.1$ & $72.5 \pm 0.7$ & $78.6 \pm 0.3$ & $77.1 \pm 1.3$ & $79.0 \pm 0.3$ & $86.5 \pm 0.6$ & $87.8 \pm 1.4$ \\
ARMA & $82.8 \pm 0.6$ & $89.8 \pm 0.1$ & $88.2 \pm 1.0$ & $72.3 \pm 1.1$ & $79.9 \pm 0.6$ & $76.7 \pm 1.5$ & $78.8 \pm 0.3$ & $88.1 \pm 0.2$ & $88.7 \pm 1.0$ \\
APPNP & $83.3 \pm 0.1$ & $90.4 \pm 0.2$ & $87.5 \pm 1.4$ & $71.8 \pm 0.4$ & $79.2 \pm 0.4$ & $77.3 \pm 1.6$ & $80.2 \pm 0.2$ & $87.4 \pm 0.3$ & $88.2 \pm 1.1$ \\
HGCN & $79.8 \pm 1.2$ & $89.7 \pm 0.4$ & $87.7 \pm 1.1$ & $70.0 \pm 1.3$ & $79.2 \pm 0.5$ & $76.9 \pm 1.3$ & $78.4 \pm 0.6$ & $88.0 \pm 0.5$ & $88.0 \pm 1.6$ \\
\hline GraphNAS-R & $83.3 \pm 0.4$ & $90.0 \pm 0.3$ & $88.5 \pm 1.0$ & $73.4 \pm 0.4$ & $81.1 \pm 0.3$ & $76.5 \pm 1.3$ & $79.0 \pm 0.4$ & $90.7 \pm 0.6$ & $90.3 \pm 0.8$ \\
GraphNAS-S & $81.4 \pm 0.6$ & $90.1 \pm 0.3$ & $88.5 \pm 1.0$ & $71.7 \pm 0.6$ & $79.6 \pm 0.5$ & $77.5 \pm 2.3$ & $79.5 \pm 0.5$ & $88.5 \pm 0.2$ & $88.5 \pm 1.1$ \\
GraphNAS & $\mathbf{8 3 . 7} \pm \mathbf{0 . 4}$ & $\mathbf{9 0 . 6} \pm \mathbf{0 . 3}$ & $\mathbf{8 8 . 9} \pm \mathbf{1 . 2}$ & $\mathbf{7 3 . 5} \pm \mathbf{0 . 3}$ & $\mathbf{8 1 . 2} \pm \mathbf{0 . 5}$ & $\mathbf{7 7 . 6} \pm \mathbf{1 . 5}$ & $\mathbf{8 0 . 5} \pm \mathbf{0 . 3}$ & $\mathbf{9 1 . 2} \pm \mathbf{0 . 3}$ & $\mathbf{9 1 . 1} \pm \mathbf{1 . 0}$ \\
\hline
\end{tabular}

Table 3: Node classification results w.r.t. accuracy, where "semi" stands for semi-supervised learning experiments, "sup" for supervised learning experiments and "rand" for supervised learning experiments with randomly split data.

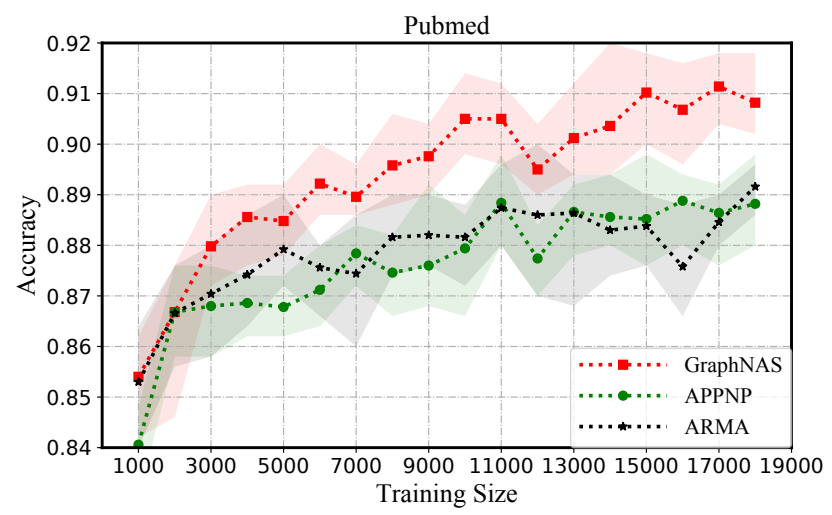

Figure 3: Comparisons w.r.t. the size of training data. When the training size exceeds 3,000, the model designed by GraphNAS always outperforms ARMA and APPNP.

Measures. We compare architectures designed by GraphNAS with the state-of-the-art GNNs, such as GCN, GAT, ARMA, APPNP [Klicpera et al., 2019] and HGCN [Hu et al., 2019], on node classification tasks. We use accuracy as the measure for comparison. All the results are the average scores for 100 runs with different random seeds.

\subsection{Parameter Settings}

The GNN architectures used in GraphNAS are implemented by PYG [Fey and Lenssen, 2019].

Hyper-parameters of the controller: The controller is a onelayer LSTM with 100 hidden units. It is trained with the ADAM optimizer with a learning rate of 0.00035 . The weights of the controller are initialized uniformly between -0.1 and 0.1 . To prevent premature convergence, we also use a tanh of 2.5 and a temperature of 5.0 for the sampling logits [Bello et al., 2017], and add the controller's sample entropy to the reward, weighted by 0.0001 . After GraphNAS searches $S=2000$ architectures, we collect the top $K=5$ architectures that achieve the best validation accuracy. Then we train those model for $N=20$ times to choose the best models. Each GNN designed by GraphNAS contains $L=2$ layers for fair comparisons.

Hyper-parameters of GNNs: Once the controller samples an architecture, a child model is constructed and trained for 300 epochs. We apply the L2 regularization with $\lambda=0.0005$,

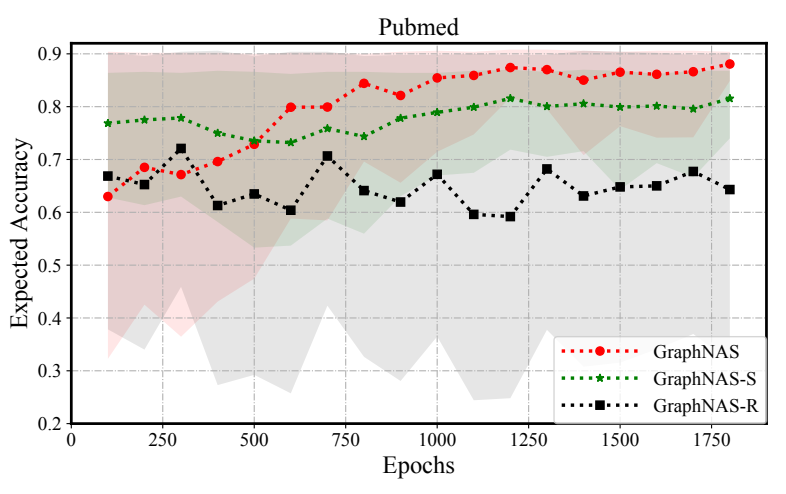

Figure 4: Comparisons w.r.t. the number of search epochs on Pubmed. The expected accuracy of the architecture designed by GraphNAS raises with the search epochs. GraphNAS outperforms Simple-NAS after 500 epochs.

dropout probability $p=0.6$, and learning rate $l r=0.005$ as the default parameters. To achieve the best results, the hyper-parameters of the GNN models are searched over the following search space:

- Hidden size: $[8,16,32,64,128,256,512]$

- Learning rate: [1e-2, 1e-3, 1e-4, 5e-3, 5e-4]

- Dropout: $[0.0,0.1,0.2,0.3,0.4,0.5,0.6,0.7,0.8,0.9]$

- $L_{2}$ regularization strength: [0, 1e-3, 1e-4, 1e-5, 5e-5, 5e-4]

For GraphNAS, the hyper-parameters are predicted by the controller. While for the other models, the hyper-parameters is optimized by hyperopt ${ }^{2}$.

\subsection{Results of Node Classification}

To validate the performance of node classification, we compare the models designed by GraphNAS with the benchmark GNNs in semi-supervised task and supervised task. The performance in terms of accuracy is shown in Table 3. The best performance of each column is highlighted with boldface.

In the semi-supervised learning task, the datasets follow the settings of [Kipf and Welling, 2017]. During training, only 20 labels per class are used for each citation network, 500 nodes in total for validation and 1,000 nodes for testing.

${ }^{2}$ https://github.com/hyperopt/hyperopt 


\begin{tabular}{c|cccc}
\hline Model & CS & Physics & Computers & Photo \\
\hline GCN & $95.5 \pm 0.3$ & $98.3 \pm 0.2$ & $88.0 \pm 0.6$ & $95.4 \pm 0.3$ \\
GAT & $95.5 \pm 0.3$ & $98.1 \pm 0.2$ & $89.1 \pm 0.6$ & $95.6 \pm 0.3$ \\
ARMA & $95.4 \pm 0.2$ & $\mathbf{9 8 . 5} \pm \mathbf{0 . 1}$ & $86.1 \pm 1.0$ & $94.8 \pm 0.8$ \\
APPNP & $95.6 \pm 0.2$ & $\mathbf{9 8 . 5} \pm \mathbf{0 . 1}$ & $89.8 \pm 0.4$ & $95.8 \pm 0.3$ \\
\hline GraphNAS & $\mathbf{9 7 . 1} \pm \mathbf{0 . 2}$ & $\mathbf{9 8 . 5} \pm \mathbf{0 . 2}$ & $\mathbf{9 2 . 0} \pm \mathbf{0 . 4}$ & $\mathbf{9 6 . 5} \pm \mathbf{0 . 4}$ \\
\hline
\end{tabular}

Table 4: Transferring architectures designed by GraphNAS on the citation networks to the other four datasets

In the supervised learning task, 500 nodes in each dataset are selected as the validation set and 500 nodes are selected as the test set. The rest of nodes are selected from the graph as training data. The architectures designed by GraphNAS are showed in Figure 5.

The work [Shchur et al., 2018] claims that data split has an impact on the performance. Thus, we randomly split the datasets for 100 times to verify the models designed in the supervised learning task. Each split contains 500 nodes for evaluation, 500 nodes for test, and the rest for training.

From Table 3, we observe that the models designed by GraphNAS rival the best human-invented architectures. The model designed by GraphNAS always outperforms the stateof-the-art. The average improvement of GraphNAS is reasonable when the training data is small, such as on Cora and Citeseer. Moreover, GraphNAS performs significantly better than others when the number of training data is large, such as on Pubmed. Overall, the improvement of the model designed by GraphNAS on Pubmed is $3.1 \%$ in terms of accuracy.

The size of training data. In this part, we test the performance with respect to the number of training data. We split the training data ranging from 1,000 to 18,000 . The results are shown in Figure 3. From the results, we observe that the model designed by GraphNAS always outperforms ARMA and APPNP when the number of training nodes exceeds 3,000 .

\subsection{Results of Transfer Learning}

In this part, we apply the architectures discovered by GraphNAS on the citation networks to supervised node classification on different data sets, such as the coauthor networks MS-CS and MS-Physics, and the product networks of Amazon Computers and Amazon Photo. As shown in Table 4, the models designed by GraphNAS can obtain competitive results when transferred to new datasets.

\subsection{Variants of GraphNAS}

Based on the original GraphNAS, we construct two variants: GraphNAS-R that randomly selects graph neural network architectures from a given search space, and GraphNAS-S that simply searches for an entire graph neural architecture where each layer containing only one computational node and take the last layer as input.

Tables 3 presents the results of the architectures designed by the two variants. We observe that the combination of existing graph neural operators may achieve better results on bigger networks. The comparisons between GraphNAS-R and GraphNAS show that the search space we defined is reason-

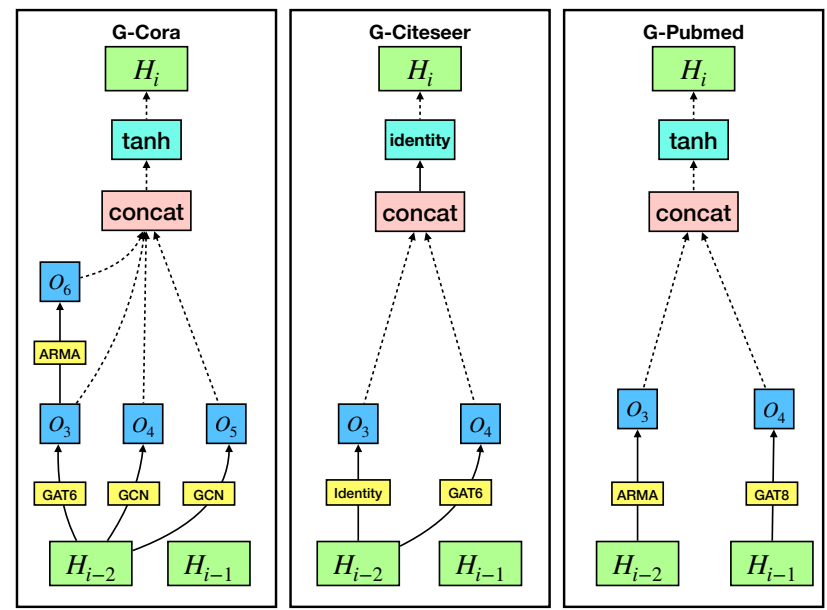

Figure 5: An example of the graph neural architectures designed by GraphNAS on the supervised learning task. The architecture $G$-Cora designed by GraphNAS on Cora is $[1$, gat_6, 1, gcn, 1, gcn, 3 , arma,tanh, concat $]$, the architecture $G$-Citeseer designed by GraphNAS on Citeseer is $[1$, identity, 1, gat_6, identity, concat $]$, and the architecture G-Pubmed designed by GraphNAS on Pubmed is $[2$, gat_8, 1, arma, tanh, concat $]$.

able, and the search algorithm converges to the optimal solution.

In order to verify the effectiveness of the search algorithm with reinforcement learning, we compare the expected validation accuracy over time during the architecture search, as shown in Figure 4.

In terms of computation time, for supervised learning the search time on Pubmed is 12 GPU hours, Cora and Citeseer 6 GPU hours. For semi-supervised learning, the search time on Pubmed is 9 GPU hours, Cora and Citeseer 2 GPU hours. The experiments are tested on a single NVIDIA 1080Ti.

\section{Conclusions}

In this paper, we study the challenging problem of graph neural architecture search using reinforcement learning. We present a new model GraphNAS which can automatically design the best graph neural architectures. A new search space is designed to include the operators from the state-of-the-art GNNs, and a policy gradient algorithm is used to iteratively solve the learning. Experiment results on real-world datasets show that GraphNAS can design a novel network architecture that rivals the best human-invented architecture in terms of validation set accuracy. Moreover, We release the python codes on Github for comparison.

\section{Acknowledgements}

This work was supported in part by the National Key Research and Development Program of China (No. 2017YFB0803300), the NSFC (No. 61872360), the Youth Innovation Promotion Association CAS (No. 2017210), and an Australian Government Research Training Program Scholarship. 


\section{References}

[Bello et al., 2017] Irwan Bello, Barret Zoph, Vijay Vasudevan, and Quoc V. Le. Neural optimizer search with reinforcement learning. In ICML, 2017.

[Bianchi et al., 2019] Filippo Maria Bianchi, Daniele Grattarola, Lorenzo Livi, and Cesare Alippi. Graph neural networks with convolutional arma filters. ArXiv, abs/1901.01343, 2019.

[Cai et al., 2019] Han Cai, Ligeng Zhu, and Song Han. ProxylessNAS: Direct neural architecture search on target task and hardware. In International Conference on Learning Representations, 2019.

[Defferrard et al., 2016] Michaël Defferrard, Xavier Bresson, and Pierre Vandergheynst. Convolutional neural networks on graphs with fast localized spectral filtering. In NIPS, 2016.

[Dong and Yang, 2019] Xuanyi Dong and Yi Yang. Searching for a robust neural architecture in four gpu hours. In Proceedings of the IEEE Conference on Computer Vision and Pattern Recognition (CVPR), pages 1761-1770, 2019.

[Fey and Lenssen, 2019] Matthias Fey and Jan E. Lenssen. Fast graph representation learning with PyTorch Geometric. In ICLR Workshop on Representation Learning on Graphs and Manifolds, 2019.

[Gori et al., 2005] M. Gori, G. Monfardini, and F. Scarselli. A new model for learning in graph domains. In IEEE International Joint Conference on Neural Networks, 2005.

[Hamilton et al., 2017] William L. Hamilton, Zhitao Ying, and Jure Leskovec. Inductive representation learning on large graphs. In NIPS, 2017.

[Hu et al., 2019] Fenyu Hu, Yanqiao Zhu, Shu Wu, Liang Wang, and Tieniu Tan. Hierarchical graph convolutional networks for semi-supervised node classification. In Proceedings of the Twenty-Eighth International Joint Conference on Artificial Intelligence, (IJCAI), 2019.

[Kipf and Welling, 2017] Thomas N. Kipf and Max Welling. Semi-supervised classification with graph convolutional networks. In International Conference on Learning Representations (ICLR), 2017.

[Klicpera et al., 2019] Johannes Klicpera, Aleksandar Bojchevski, and Stephan Günnemann. Combining neural networks with personalized pagerank for classification on graphs. In International Conference on Learning Representations, 2019.

[Liu et al., 2018a] Hanxiao Liu, Karen Simonyan, and Yiming Yang. Darts: Differentiable architecture search. CoRR, abs/1806.09055, 2018.

[Liu et al., 2018b] Ziqi Liu, Chaochao Chen, Longfei Li, Jun Zhou, Xiaolong Li, and Le Song. Geniepath: Graph neural networks with adaptive receptive paths. CoRR, abs/1802.00910, 2018.

[Niepert et al., 2016] Mathias Niepert, Mohamed Ahmed, and Konstantin Kutzkov. Learning convolutional neural networks for graphs. In ICML, 2016.
[Pham et al., 2018] Hieu Pham, Melody Y. Guan, Barret Zoph, Quoc V. Le, and Jeff Dean. Efficient neural architecture search via parameter sharing. In ICML, 2018.

[Real et al., 2018] Esteban Real, Alok Aggarwal, Yanping Huang, and Quoc V. Le. Regularized evolution for image classifier architecture search. CoRR, abs/1802.01548, 2018.

[Shchur et al., 2018] Oleksandr Shchur, Maximilian Mumme, Aleksandar Bojchevski, and Stephan Günnemann. Pitfalls of graph neural network evaluation. 2018.

[Vaswani et al., 2017] Ashish Vaswani, Noam Shazeer, Niki Parmar, Jakob Uszkoreit, Lawrence Jones, Aidan N. Gomez, Lukasz Kaiser, and Illia Polosukhin. Attention is all you need. In NIPS, 2017.

[Velickovic et al., 2017] Petar Velickovic, Guillem Cucurull, Arantxa Casanova, Adriana Romero, Pietro Liò, and Yoshua Bengio. Graph attention networks. CoRR, abs/1710.10903, 2017.

[Williams, 1992] Ronald J. Williams. Simple statistical gradient-following algorithms for connectionist reinforcement learning. Machine Learning, 8(3-4):229-256, 1992.

[Wu et al., 2019a] Felix Wu, Amauri H. Souza, Tianyi Zhang, Christopher Fifty, Rui Zhang, and Kilian Q. Weinberger. Simplifying graph convolutional networks. In ICML, 2019.

[Wu et al., 2019b] Shu Wu, Yuyuan Tang, Yanqiao Zhu, Liang Wang, Xing Xie, and Tieniu Tan. Session-based recommendation with graph neural networks. In $A A A I$, 2019.

[Xie et al., 2019] Sirui Xie, H P Zheng, Chunxiao Liu, and Liang Lin. Snas: Stochastic neural architecture search. In International Conference on Learning Representations, 2019.

[Xu et al., 2018] Keyulu Xu, Weihua Hu, Jure Leskovec, and Stefanie Jegelka. How powerful are graph neural networks? CoRR, abs/1810.00826, 2018.

[Yan et al., 2018] Sijie Yan, Yuanjun Xiong, and Dahua Lin. Spatial temporal graph convolutional networks for skeleton-based action recognition. In $A A A I, 2018$.

[You et al., 2019] Jiaxuan You, Rex Ying, and Jure Leskovec. Position-aware graph neural networks. In ICML, 2019.

[Yu et al., 2018] Bing Yu, Haoteng Yin, and Zhanxing Zhu. Spatio-temporal graph convolutional networks: A deep learning framework for traffic forecasting. In IJCAI, 2018.

[Zaheer et al., 2017] Manzil Zaheer, Satwik Kottur, Siamak Ravanbhakhsh, Barnabas Poczos, and Alexander Smola. Deep sets. 2017.

[Zoph and Le, 2016] Barret Zoph and Quoc V. Le. Neural architecture search with reinforcement learning. CoRR, abs/1611.01578, 2016. 